\title{
Genes, brain, and behavior: Bridging disciplines
}

\author{
JOHN A. FOSSELLA and B. J. CASEY \\ Weill Medical College of Cornell University, New York, New York
}

\begin{abstract}
With excitement surrounding the publication of the human genome, scientists have set out to uncover the functions of specific genes. This special issue on Genes, Brain, and Behavior attempts to present research strategies that connect major avenues of genetic research across disciplines. For example, anatomical information provided by brain imaging can serve as a convenient link between anatomical abnormalities seen in knockout/transgenic mouse models and abnormal patterns of brain activity seen in certain patient populations. Identifying genetic risk factors for disorders with carefully designed cognitive assays is another strategy that has gained increasing attention. These approaches are being combined with behavioral studies of mouse models of gene function. Alone, each of these approaches provides limited information on gene function in complex human behavior, but together, they are forming bridges between animal models and human psychiatric disorders.
\end{abstract}

The publication of the human genome sequence has presented researchers with new opportunities in their attempt to understand gene function. Two major avenues of research have thus opened up: human psychiatric genetics, in which genes are linked to clinical DSM-IV criteria; and mouse molecular genetics, in which genes can be linked to alterations in cell physiology and gene expression. There has, however, been some difficulty in reconciling these two avenues of research, which Hyman and Fenton (2003) have referred to as a "translational bottleneck." Recently, a number of bioinformatic tools, new methodologies, and experimental strategies have been developed that allow for the straightforward testing of hypotheses that interlock with the mouse and human data. These new tools, initiatives, and strategies are for the most part themselves untested and in need of validation. In this special issue, we present research strategies that reside in the as yet unformalized, murky passages and alleyways that connect the aforementioned avenues of genetic research.

As information accumulates on the human and mouse sides of the bottleneck, there is growing demand, and hence much opportunity, for expertise in levels of analysis that lie between the purely clinical and the purely cell biological. Insofar as neuroscientists are interested in applying genetic methods, new strategies for eliminating the bottleneck will likely arise with time. Recently, for example, genetic research has led to exciting results in the field of "imaging genetics." In principle, the anatomical information provided by brain imaging can serve as a convenient link between anatomical abnormalities seen in knockout/transgenic mouse models and abnormal patterns of brain activity seen in certain patient populations. Several authors in this issue, including Brown and Hariri

Reprint requests should be addressed to either J. A. Fossella or B. J. Casey, Sackler Institute, Weill Medical College of Cornell University, 1300 York Ave., Box 140, New York, NY 10021 (e-mail: jaf2014@med .cornell.edu or bjc2002@med.cornell.edu).
(2006); Wrase, Reimold, Puls, Kienast, and Heinz (2006); Bishop, Cohen, Fossella, Casey, and Farah (2006); and Fossella, Green, and Fan (2006) have adopted this approach, but all of them have adapted the basic genetic and imaging methods in unique ways. Another related approach is to add genetic methods to pure cognitive and neuropsychological approaches (cognitive genetics). Karmiloff-Smith (2006), Waldman et al. (2006), and Espeseth et al. (2006) are developing cognitive genetic approaches that will become increasingly essential as genetic risk factors for disorders are identified. These approaches are beginning to be constrained by behavioral studies of mouse models such as those described by Bath and Lee (2006) and Kruzich, Mitchell, Younkin, and Grandy (2006) (see Table 1 for methods described in this issue).

The impetus for this special issue stems in part from lectures and issues discussed at the John Merck Fund Summer Institute on the Biology of Developmental Disabilities. This annual course emphasizes recent developments regarding genes, brain, and behavior and is taught by distinguished international scientists with expertise in the areas of psychology, developmental neurobiology, neuroscience, and molecular biology. ${ }^{1}$ The goal of the course is to introduce junior investigators to genetic, imaging, computational, and behavioral methods for constraining theories and interpretations of typical and atypical behavior. The goal of this special issue is similar. The objective is to provide a framework within which future genetic studies can be constrained and evaluated for validity and relevance with respect to existing data with a converging methods approach. These methods include the use of cognitive and neuroimaging genetics, together with mouse models of gene function. A review of basic genetic terms and bioinformatics may be useful in introducing these approaches to new readers of the genetic literature.

\section{Genetic Terms and Bioinformatics}

Genomes, genes, and SNPs. The term gene refers to a portion of the human genome that contains the instruc- 
Table 1

Current Approaches for Examining Somewhat Controversial and

Simple Associations Among Genes, Behaviors, and Brain Regions

\begin{tabular}{|c|c|c|c|}
\hline Approaches & Genes & Behaviors & Brain Regions \\
\hline \multicolumn{4}{|l|}{ Cognitive Genetics } \\
\hline \multirow[t]{2}{*}{ Karmiloff-Smith } & $F O X P 2$ & vocal learning & basal ganglia \\
\hline & $L I M K 1$ & spatial deficits? & parietal cortex, whole brain \\
\hline Waldman et al. & $A D R A 2$ & executive function & prefrontal cortex \\
\hline Espeseth et al. & $A P O E$ and $n A C h R$ & attention/orienting & parietal cortex, white matter \\
\hline \multicolumn{4}{|l|}{ Imaging Genetics } \\
\hline Brown \& Hariri & SERT & emotion processing & amygdala \\
\hline Wrase et al. & SERT & anxiety, aggression & raphe nucleus \\
\hline Bishop et al. & $M A O A$ & affect regulation & orbital and prefrontal cortex \\
\hline Fossella et al. & DRD2 & attentional conflict & anterior cingulate cortex \\
\hline \multicolumn{4}{|l|}{ Mouse Models } \\
\hline Bath \& Lee & $B D N F$ & contextual learning & hippocampus \\
\hline Kruzich et al. & DRD2 & reversal learning & striatum \\
\hline
\end{tabular}

tions for the production of a protein or ribonucleic acid (RNA). The Human Genome Project led to the recent publication of the sequence of a sample human genome (the full deoxyribonucleic acid [DNA] sequence of an organism) that reveals approximately 25,000 genes. The human genome sequence is just one of many complete genome sequences now available, including those for the chimpanzee, elephant, dog, rat, shrew, chicken, pufferfish, rabbit, and fruit fly, as well as an inordinate number of bacteria, fungi, and nematodes (see www.genome.gov/10002154).

The studies reported in this issue, and most genetic studies using intermediate levels of analysis, are designed not from a whole-genome point of view, but rather from the researcher's having selected from the genome, at the outset, a so-called candidate gene. The candidate gene study design is appropriate when substantial amounts of converging evidence suggest that a particular gene contributes to heritable variation or risk (i.e., the extent to which variation in a trait among members of a population is determined by inherited genetic variation). In the past, the selection of a candidate gene represented, very simply, an educated guess about what gene might be involved in the trait of interest. For example, in behavioral genetics, many candidate genes were chosen on the basis of pharmacological studies. Dopamine receptors and metabolic enzymes have been widely explored in genetic studies on working memory, attention, and various psychiatric disorders.

Recently, bioinformatic tools have become increasingly available for hypothesis generation and data interpretation of candidate gene studies. ${ }^{2}$ Bioinformatic databases and data mining tools are supported and distributed by multidisciplinary research groups consisting of computer scientists, molecular biologists, mathematicians, biochemists, research physicians, and structural biologists. Such tools are particularly valuable in linking mouse genetic data and human genetic data. In most, if not all, Internetbased genome browsers, investigators can easily query the structure, sequence, and many other attributes of a candidate gene or chromosomal region.

For candidate gene association studies, the individual nucleotides that vary from person to person are most im- portant. These variable sites arise from errors in genome replication, which in humans are rare; but they occur often enough in our genome of 3 billion nucleotides for approximately 100 base pair changes to accumulate per generation per genome. Most of these minor base pair changes are lost over time as generations come and go. However, some of these changes continue to be passed on and even spread across populations. These ancient DNA changes, known as polymorphisms or single nucleotide polymorphisms (SNPs) are presently found in slightly more than 1 out of every 1,000 nucleotides. These changes then lead to an enormous amount of DNA sequence variability across human populations.

The sequencing of multiple human genomes from disparate cultures and geographic regions has produced a catalogue of human genetic variability (see www.genome .gov/17015412). NCBI's official site, known as dbSNP, contains more than 17 million raw human entries that coalesce into about 9 million unique entries, of which half have been validated. Investigators who wish to explore whether specific genetic variants are associated with a specific trait (e.g., performance on a cognitive task) or an affected group of patients can use these databases to get an overall snapshot of the possible polymorphic sites of interest in a given gene. Comparisons of gene expression between individuals or experimental groups has been greatly facilitated in the past decade by the introduction of genome-wide microarrays or gene chips. Proprietary (e.g., Affymetrix) and nonproprietary technology is now available that permits the single-use assay of the expression of thousands of candidate genes in parallel.

Population stratification. One of the most critical issues in designing candidate gene association studies is a consideration of the frequency of the polymorphic alleles under investigation. Allele frequencies can be extremely low, and they vary with population. For example, the frequency of the E4 allele of Apolipoprotein E (APOE) ranges from 5\% in Taiwan and Sardinia to $40 \%$ in Pygmies (Corbo \& Scacchi, 1999; Siest et al., 1995). It is desirable to choose a polymorphism whose alternate alleles exist at relatively high frequencies within and across populations. 
Choosing an allele that is present in only $5 \%$ of the population creates practical difficulties from a recruitment perspective, but it also makes interpretation problematic.

The Human Genome Diversity Project has compared sequences of individuals from Africa, Asia, Europe, Pacifica and the Americas. Sequence comparisons across human populations reveal that variations in the sequence of a gene occur several times within any given gene and that each person carries a genome sequence that is approximately $0.1 \%$ different from that of any other human (available at www.cshl.snp.org). These differences in the sequence of a gene allow the different gene variants to be subclassified into alleles. An allele is one member of a pair or series of genes that occupy a specific position on a specific chromosome. Not surprisingly, when sampling human populations from disparate geographic and racial/ ethnic groups, one finds that there are dramatic differences in the type and frequency of allelic variation.

A convenient framework for understanding such differences can be formed by considering the natural history of Homo sapiens. In the course of human history, errors in the replication of chromosomal DNA have been rare, but they occur often enough in our genome of 3 billion nucleotides for hundreds of independent base pair changes to accumulate per generation per genome (Nachman \& Crowell, 2000). Although many of these minor base pair changes have been lost over time as local mating groups have thrived and crashed, by chance some changes have persisted in local populations and even, perhaps due to natural selection, spread across populations. The fragmented way in which this genetic variation is geographically distributed has its origins in the migratory patterns of human populations as far back as 10,000 or even 60,000 years ago. As the first human populations migrated out of Africa and populated Asia, Europe, and the Americas, differences in culture, climate, and landscape served as natural barriers to prevent the intermating of local populations. The present-day collection of human polymorphisms itself (found at dbSNP and elsewhere) is, then, both a static catalogue of human genetic variability and also a history book that contains details about the emergence of migratory routes of humans.

In genetic research today, investigators may be unaware of an individual's ancestral lineage, but they have long been aware of its importance and often ask patients to selfidentify with a few major ethnic groups. Typically, attributes of an individual's culture are shaped by local climate and geography and hence can be tied historically to ancestral migratory routes. In efforts to understand behavior, an understanding of the relationship between genetic variation and self-reported ethnic and cultural attributes will be an important factor to examine. In sum, because allele frequencies can vary with population, ancestral heritage, and population admixture, it is important to monitor these, in order to avoid spurious results in studies of associations between a polymorphism and a trait.

Trait validity and reliability. One basic goal of genetic studies is simply to understand aspects of physical or behavioral variation in biological systems that are inherited - that is, passed on from parents to offspring via the male and female gametes. For example, variation in skin, eye, and hair color can be ascribed to genes that encode proteins that synthesize various pigment molecules. So far, in humans, the feat of linking variation in a physical trait with a portion of the genome has been accomplished about 1,800 times (see www.ncbi.nlm.nih.gov:80/Omim/ mimstats.html for details).

Many of the articles in this issue refer to traits as endophenotypes (e.g., Waldman et al., 2006). Clarification of the use of this term may be helpful at this point. An endophenotype is similar to a psychological construct (e.g., intelligence, executive functions) in that it is not directly observable, unlike a phenotype, which refers to visible characteristics such as eye color. Endophenotypes may include biological measures that index the products of riskinducing genes or cognitive constructs thought to reflect brain mechanisms that underlie the disorder. In contrast, the genotype refers to the genetic makeup of an organism (i.e., combination of alleles), as opposed to its physical characteristics (phenotype).

Variation in complex traits such as emotion, attention, and memory can be inherited to some extent, but the genes that encode proteins that regulate the neural development of structures involved in behavior are poorly understood. The genetics of skin, eye, and hair color are straightforward, in part because there is little dispute about how to measure the trait and how to define the relevant aspects of a measurement scale (e.g., blue, green, hazel, or brown eyes). If our interest is to understand how genes influence the development of the emotional system, however, it is not so clear how to define the relevant traits and how to make measurements that are both valid and reliable. Therefore, it is important to establish the reliability of the measurement so that within-subjects variation is minimal compared to between-subjects variation.

Trait heritability. Only after an assay has been developed that reliably captures variation in a valid trait can one jump to genetic research to ask whether such variation is underlain by genetic factors. For example, tall parents frequently produce tall children, but variation in height among humans is not accounted for by parental genes alone, since diet, exercise and sleep regimens can influence a child's stature. Heritability $\left(h^{2}\right)$ is a term that describes the extent to which variation in a trait (e.g., cognitive performance) among members of a population is determined by inherited genetic variation. Heritability can vary from 1 (high) to 0 (low) and can be estimated in a number of ways (e.g., family, twin, and adoption studies).

The most standard approach for estimating heritability is to compare the correlation in performance of identical (monozygotic, or MZ) twins relative to fraternal (dizygotic, or DZ) twins. Since MZ twins have a genome of identical sequence, and DZ twins have a genome that is about 50\% identical, higher correlation among MZ twins suggests that genetic factors contribute significantly to the variation among individuals for that trait, but the very 
similar environment in which twins are raised likely contributes as well. A heritability index can be computed from the differences between MZ and DZ correlations. In this way, it has been shown that performance on many commonly used cognitive tasks is influenced by genetic factors.

A number of behavioral genetic studies have begun to examine the heritability of cognitive performance across a wide number of tasks (Bartfai, Pedersen, Asarnow, \& Schalling, 1991; Cornblatt, Risch, Faris, Friedman, \& ErlenmeyerKimling, 1988; Myles-Worsley \& Coon, 1997). For example, using the continuous performance task (CPT), Cornblatt et al. (1988) have shown that CPT performance as measured by $d^{\prime}$ has an estimated heritability among normal subjects of .49. The span of apprehension task (SPAN), a visual search task, has been shown to have a heritability among normal subjects of .65 (Bartfai et al., 1991). Studies of twins show that spatial working memory, divided attention, choice reaction time, and selective attention (Cannon et al., 2000); attentional set-shifting (Pardo et al., 2000); sensorimotor gating (Geyer \& Braff, 1987); smooth pursuit eye tracking (Katsanis, Taylor, Iacono, \& Hammer, 2000); and executive attention (Fan, Wu, Fossella, \& Posner, 2001) are all underlain by inherited factors. In parallel to behavioral genetic studies, anatomical studies in rodents, nonhuman primates, and humans have begun to examine genetic determinants of brain size (Cheverud et al., 1990; Thompson, Cannon, \& Toga, 2002) and structural aspects of specific brain regions such as the frontal cortex (Thompson et al., 2000; Tramo et al., 1998) and corpus callosum (Oppenheim, Skerry, Tramo, \& Gazzaniga, 1989).

Functionality. Two basic questions can arise when one considers how to explain the heritability of the trait of interest in terms of the 25,000 possible genes in the genome. Two questions that might arise are, "How do individuals with and without the trait differ in the sequence of their genomes?" and "How do individuals with and without the trait differ in the expression of their genomes?" For example, in investigating a candidate gene, it is useful to understand how to classify variable sites according to functionality and frequency. Some variable sites lie within a gene and affect the structure of the protein (e.g., brainderived neurotrophic factor or BDNF and the catechol $O$-methylase or COMT genes). Other sites lie outside the coding sequence, yet they are able to influence the amount of expression of the encoded protein (serotonin transporter or SERT gene). Still other sites lie inside or outside a protein-coding region yet have no effect at all on the encoded protein structure or expression. Unfortunately, many gene association studies are carried out using such nonfunctional polymorphic sites (see Fossella et al., 2006, for discussion).

\section{Putting Genetic Variant and Phenotype Together: What Are These Genes Doing?}

The basic principles involving the validity, reliability, heritability, and functionality of the basic gene association design may help narrow the focus of investigators and promote studies in the manner described by Brown and Hariri (2006). These investigators have set an example for experimental replication as well as for follow-up studies that examine neural mechanisms. Below we provide examples of attempts to link genes, brain, and behavior using cognitive genetics, imaging genetics, and mouse models of behavior. Each of these approaches alone provides limited information on gene function in complex human behavior, but together they are forming bridges between animal models and human psychiatric disorders.

It is impossible to specify which of the current methodological alleyways will evolve into established thoroughfares. At present, there are at least 25,000 known genes in the human/mouse mammalian genome. Within each gene there are anywhere from 10 to 100 variable sites across the human diaspora. Within each individual is a brain that can be measured at the $1-3 \mathrm{~mm}^{3}$ voxel level, whose dynamic networks are capable of an infinite variety of computational states, moods, and behaviors. Clearly, one-to-one statistical mapping of genetic variants to functional voxels, cognitive states, or performance measures, while a convenient first step, could be performed ad infinitum.

The challenge of linking genes, brain, and behavior becomes even more daunting when we consider gene function in a dynamically changing system, such as the developing one. We begin this special issue with a cautionary paper by Karmiloff-Smith (2006) that addresses complexities that arise when one interprets simple gene-behavior associations in the context of development. KarmiloffSmith argues that in the excitement to use the human genome project to uncover the functions of specific genes, researchers often ignore a fundamental factor: the gradual process of ontogenetic development. Claims about genebehavior relations are typically based on a phenotypic end state and grounded in the neuropsychological tradition of studying adults whose brains are fully and normally developed until a brain insult later in life. The developing brain is of course different, in that brain regions and circuitry are not specialized at birth. Many years are required for the specialization of neural networks as a result of complex interaction with the environment in gene expression and the resulting phenotype. Karmiloff-Smith argues that "because ontogenetic development and timing play such important roles in development, a tiny impairment in the start state of the brain of a child with a genetic disorder may affect several brain regions, some more profoundly and others more subtly, giving rise in the phenotypic endstate to what appears to be a domain-specific outcome" (p. $\mathrm{xxx}$ ). It is important to keep this neuroconstructivist perspective in mind when one is interpreting simple genebehavior associations in developmental disorders such as those described by Waldman et al. (2006) and by Espeseth et al. (2006) in this issue.

Cognitive genetics. A new direction in candidate gene and gene association research is the emphasis on more precise phenotypes. Traditionally, psychiatric diagnosis or neuropsychological measures of general function have 
been used. The complexities of such phenotypes make it difficult to link any one gene or combination of genes to a behavior or cluster of behaviors. Without precise measures, the trait of interest will lack validity.

Karmiloff-Smith (2006) illustrates the precision with which behavioral traits must be assayed, in her studies of Williams syndrome and the domain of face processing. Several investigators have claimed that face-processing in individuals with Williams syndrome is intact, while spatial processing is impaired (e.g., Bellugi, Wang, \& Jernigan, 1994). Although people with Williams syndrome fall in the normal range in performance of some standardized face processing tasks like the Benton (Rossen, Jones, Wang, \& Klima, 1995), the means by which they achieve this success turns out to be very different from that for controls.

Karmiloff-Smith and colleagues have shown that whereas typically developing children use configural processing, the individuals with Williams syndrome use other types of processing (e.g., featural). When comparing brain processing of human faces, monkey faces, and cars as measured by high-density event related potentials, it was found that typical controls displayed a strong N170 (an early electrophysiological index of face processing) for human and monkey faces, with no such increase in amplitude for the brain processing of cars. By contrast, individuals with Williams syndrome showed a reduced N170, which was similar for all three classes of stimuli. In other words, for the Williams syndrome group, faces were processed in much the same way as were cars, without evidence for specialization of function. It is not the case, then, that individuals with Williams syndrome have an intact face processing module and an impaired spatial module as suggested by general neuropsychological assessments. Both domains of behavior appear to be impaired rather than a face or spatial processing impairment per se. This paper underscores the importance of valid behavioral assays in characterizing phenotypes of developmental disorders.

Waldman et al. (2006) also use a cognitive genetic approach to test for associations between executive function measures and a candidate gene implicated in prefrontal function in individuals with ADHD. They first studied the validity of executive functions as an endophenotype for ADHD by examining the association between executive function measures and ADHD symptoms. Symptoms and measures were assessed in approximately 200 probands (individuals or members of a family being studied in a genetic investigation) and their unaffected siblings relative to non-ADHD controls and their correlation in siblings. The one measure that met all the validity criteria for an endophenotype was mean reaction time on the stop signal task. Validity criteria that were met with this measure included (1) performance that was correlated with ADHD symptoms; (2) greater deficits in ADHD probands than in their unaffected siblings; (3) greater deficits in unaffected siblings than in nondisordered controls; and (4) performance that was moderately correlated in the siblings.
After establishing the validity of executive function as an endophenotype of ADHD, Waldman et al. (2006) tested for associations between executive function measures and the $\alpha 2$ noradrenergic receptor gene (i.e., $A D R A 2 A$ ) in individuals with ADHD. The focus on this candidate gene was based on the link between the Alpha2-class of adrenergic receptors in noradrenergic activity in prefrontal executive functions (Arnsten, 2000; Arnsten, Steere, \& Hunt, 1996). Several executive function measures (stop signal, trails, Stroop, and tower of London tasks) showed association with $A D R A 2 A$. However, these measures were shown to moderate, rather than mediate the association between ADRA2A and ADHD. Mediation refers to the extent to which an endophenotype explains part or all of the association between a genetic polymorphism and disorder (ADHD in this case) and is indicated when the association between the two is weakened or eliminated with the inclusion of the endophenotype (executive function measures) in the logistic regression equation. In contrast, moderation refers to the extent to which an endophenotype strengthens or weakens the association and is indicated by a significant interaction between the polymorphism and the endophenotype in predicting diagnostic status. In this study, executive function was not found to mediate the association between the phenotype of ADHD and the genotype of ADRA2A.

In the article by Espeseth et al. (2006), genes associated with variation in attention and neuroanatomic measures are examined. Specifically, they examine agerelated white matter and attention changes as an interaction between genes involved in efficiency of neuronal repair $(A P O E)$ and efficiency of cholinergic neurotransmission (CHRNA4). Over 200 middle-aged and older adults were genotyped and tested on a visual spatial task. $A P O E$ interacted with CHRNA4 in such a way that $A P O E^{*} 4$ carriers who were also CHRNA4 TT homozygotes showed disproportionately slowed reaction time following invalid location cues. There was a trend for individuals with combined $A P O E^{*} 4 / C H R N A 4$ TT genotypes to show both smaller white matter volume and slower overall mean reaction times on the attention task. Espeseth and colleagues conclude that the interaction of a neurotransmission gene (CHRNA4) and a susceptibility gene (APOE) suggests that the efficiency of neuronal repair mechanisms modulates the cholinergic system to influence attentional function but does not mediate the behavior.

Across all of these cognitive genetic studies, the validity and precision in measurement of the trait are emphasized. Furthermore, these studies emphasize the need to consider complex interactions among genes and the environment with development. Placing such constraints on genetic research will no doubt help to move the field away from simple one-to-one mappings of genes to behavior to more complex examination of interactions among genes and between genes and the environment in explaining behavior.

Imaging genetics. Technological advances in brain imaging techniques such as blood oxygenation level dependent functional magnetic resonance imaging (fMRI) have 
allowed researchers to noninvasively assay brain function within discrete brain circuits supporting specific cognitive and emotional processes. Genetic influences on behavior are mediated by their molecular and cellular effects on brain development and function. The functional effects of candidate variants on brain structure and function may be more readily measurable than the cognitive and emotional processes supported by these substrates. Thus, functional polymorphisms in genes weakly related to behaviors or psychiatric syndromes may be more strongly related to the function of specific neural systems. Examining genetic effects on the brain represents a critical step in understanding their ultimate contribution to variability in behavior.

Brown and Hariri (2006) describe functional neuroimaging studies that illustrate how a promoter polymorphism in the human serotonin transporter gene, which has been weakly related to several dimensions of emotional behaviors (such as neuroticism and anxiety traits), is strongly related to the engagement of neural systems - namely, the amygdala and subgenual prefrontal cortex - subserving emotional information processing. Their article provides an overview of the experimental strategy for exploring these genetic effects on brain function. They highlight the effectiveness of this strategy in delineating biological pathways and mechanisms that contribute to the emergence of individual differences in brain function, differences that potentially bias both behavior and risk for psychiatric illness.

In a complementary article, Wrase et al. (2006) show the importance of examining animal models of the serotonin transporter gene in order to understand its impact on serotonin transporter availability. This article, more than any other, also highlights the importance of experience and the environment for gene function. Identification of gene-environment interactions has become increasingly important for understanding psychiatric disorders. They review the literature on studies in nonhuman primates that assess the interaction between the genetic constitution of the regulatory region of the serotonin (5-HTT) transporter and environmental factors (e.g., stressors). Studies of nonhuman primates that have undergone social stress have shown a reduction of the serotonin turnover rate among carriers of one or two short alleles in a functional polymorphism of the 5-HTT promoter. In these primates, brain imaging studies have shown a relative increase in the availability of raphe serotonin transporters. Behaviorally, a low serotonin turnover rate and a high availability of serotonin transporters were associated with increased anxiety, impulsive aggression, and a reduced response to alcohol intake (i.e., excessive alcohol consumption). These studies highlight the importance of assessing gene-environment interactions in understanding the role of genes in behavior and risk for psychiatric illness.

Early studies of genetic effects on brain activity have primarily investigated the influence of polymorphisms in the serotonin transporter gene (SERT) on the amygdala response (see Brown \& Hariri, 2006) to threatening stimuli and dopaminergic genes, such as catechol
$O$-methyltransferase (COMT), or on prefrontal cognitive processes (e.g., working memory). In the article by Bishop et al. (2006), genetic influences on the neural systems underlying cognitive-affective interactions are examined. They focus on the COMT val158met polymorphism that has been shown to affect dopaminergic function in the prefrontal cortex, and they examine the effects of val allele load (met/met, val/met, or val/val alleles) on brain activity during attentional control under emotional distraction. They found that val load correlated positively with activity in control-related prefrontal regions and task-related regions. These findings provide an initial step toward identifying genetic contributions to betweenindividuals variability in recruitment of mechanisms that regulate affective processing.

Fossella et al. (2006) provide a cautionary example of how simple gene-behavior or gene-imaging associations can be overinterpreted. They examine a well-studied genetic polymorphism, TaqIA, which resides downstream from the dopamine D2 receptor (DRD2) gene. A great deal of electrophysiological and PET imaging data have been collected in animal and human populations that carry various genetic variants of DRD2. Only recently has it been shown that this polymorphism lies $10 \mathrm{~kb}$ downstream of the coding region of DRD2 and occurs in exon 8 of the novel kinase gene, named ankyrin repeat and kinase domain containing 1 (ANKK1) gene, resulting in a glutamate-to-lysine $(\mathrm{E} 713 \mathrm{~K})$ substitution within the eleventh ankyrin repeat. As yet, expression of the ANKK1 gene has not been detected in the developing or adult mammalian brain. Thus it is surprising that so many gene association studies have been reported for a polymorphism for this gene. Fossella et al., in describing a number of their seminal cognitive and imaging genetic studies of different attentional networks, show how they find an association between this genetic variant and patterns of brain activity too. Clearly, future studies will be needed in order to understand how a polymorphism that lies downstream of the coding region of a gene and in a gene as yet undetectable in the developing and adult mammalian brain can affect brain and behavior. One explanation for the existing literature and their finding would be that the TaqIA polymorphism in ANKK1 is in linkage disequilibrium with upstream polymorphisms in DRD2 and lies within a haplotype block that spans the overlap between the two genes. This example, however, underscores the caution that should be taken in making simple associations between brain activity and a polymorphism that is replicated and yet might be misleading in terms of the gene-behavior association. The need to examine functionality, despite replication, is thus warranted.

Mouse models. Many mouse models have begun to relate changes in gene expression to behavior. One of the most convenient approaches is to look at relationships between gene dosage and behavior. The breeding of knockout mice $(-/-)$ with wild-type mice $(+/+)$ produces heterozygote animals $(-/+)$ with one working copy of the gene of interest. Often, such changes in the amount 
of gene activity lead to behavioral variations from the behavior of the wild type. The papers in this volume by Kruzich et al. (2006) and Bath and Lee (2006) use knockout and transgenic mouse models, respectively, to understand gene function and the resulting variations in behavior.

For the reader naive to this approach, knockout mice contain the same artificially introduced mutation in every cell, abolishing the activity of a preselected gene. They are produced by a technique called gene targeting, which involves the replacement of one gene sequence, with a related sequence that has been modified to contain a mutation. The replacement occurs through a process called homologous recombination, wherein two very similar DNA sequences line up next to each other and exchange parts. Gene targeting is carried out in mouse embryonic stem cells. These cells are derived from a mouse embryo, typically male, and can therefore differentiate into all types of cells when introduced into another embryo. The aim is to get the modified embryonic stem cells to contribute to the germ line, which gives rise to sperm. Some sperm are produced that carry the desired mutation, and if they fertilize a normal egg, mice develop with one copy of the mutated gene in every cell. Interbreeding such mice will produce some homozygous individuals in the next generation; mice inheriting the mutation from both parents and therefore carrying two copies of the mutant gene are referred to as knockout mice. This resulting mutant phenotype (e.g., appearance, behavior) may provide some indication of the gene's role in the mouse, and ultimately, in humans.

A transgenic mouse contains additional, artificially introduced genetic material in every cell that can lead to a gain of function. The extra genetic material is often described as foreign DNA, but it can come from any source, including another mouse. To get the same foreign DNA sequence into every cell of the mouse, it is necessary to introduce the DNA into cells of the very young mouse embryo that will contribute to the germ line. This can be done by pronuclear microinjection, whereby the foreign DNA is introduced directly into the mouse egg just after fertilization, or by introducing the DNA into embryonic stem cells.

In the article by Bath and Lee (2006), a transgenic mouse model of brain-derived neurotrophic factor (BDNF) is described. Neurotrophins such as BDNF are a unique family of polypeptide growth factors that influence differentiation and survival of neurons in the developing nervous system. In adults, BDNF is important in regulating synaptic plasticity and connectivity in the brain. Recently, a common single nucleotide polymorphism in the human BDNF gene, resulting in a valine to methionine substitution in the prodomain (Val66Met), has been shown to lead to memory impairment and risk for psychiatric illness. An understanding of how this naturally occurring polymorphism affects behavior, anatomy, and cognition in adults is an important first step in linking genetic alterations in the neurotrophin system to definable biological outcomes in humans. Bath and Lee review the recent literature linking this BDNF polymorphism to cognitive impairment in the context of in vitro and transgenic animal studies that have established BDNF's central role in neuronal functioning in the adult brain. These studies show consistent impairments in hippocampal-dependent learning in these mice. A future direction for this research would be to exploit behavioral paradigms currently being used with these mice and adapt them for use in the imaging environment with humans, who have the BDNF (Val66Met) polymorphism. Such an approach would be a direct move toward translating the function of this gene in the context of human behavior.

The final article in this special issue describes behavioral studies performed in the dopamine $\mathrm{D}_{2}$ receptor (DRD2) knockout mouse. Kruzich et al. (2006) describe a strategy that may inform the human genetic literaturethat is, the use of gene dosage to explore behavior. Since human genetic variation is rarely equivalent to a "knockout," but rather gives rise to modest increases or decreases in gene function, the use of mice with only one functional copy of the gene more closely mimics the intermediate levels of gene expression in humans. Specifically, Kruzich et al. examine reversal learning and reinforcement efficacy in male mice lacking functional dopamine $\mathrm{D}_{2}$ receptors and their heterozygous and wild-type littermates. Their task required the mice to learn to discriminate between two odors in order to receive a food reinforcer; one odor signaled a reinforcer, whereas the other odor signaled no reinforcer. Once the mice had learned the reward contingencies, the contingencies were reversed. The number of trials required by the mice in order to relearn the new reinforcement contingencies served as an index of reversal learning. The mice lacking functional dopamine $\mathrm{D}_{2}$ receptors failed to inhibit previously reinforced responses during reversal trials. In a separate experiment, the mice were trained on a progressive ratio schedule of reinforcement. Mice lacking the functional dopamine $\mathrm{D}_{2}$ receptors earned significantly fewer reinforcers than did the heterozygous mice. These results suggest that dopamine $\mathrm{D}_{2}$ receptors may regulate reversal learning and influence the reinforcing efficacy of natural rewards. These findings are exciting for research on developmental disorders that show deficits in impulse control and addiction.

\section{Conclusions}

Given longstanding observations in the treatment of mental illness, scientists and physicians have great impetus to carry out genetic studies of brain development and function. The heritability of psychiatric illness and the heritability of response to treatment are well documented. Overwhelming evidence shows that attributes of the genome as well as interactions between the genetic material and certain attributes of the environment can influence the risk of psychiatric illness and response to treatment. More recently, technological advances in genome sequencing and genome manipulation have driven an interest in experimentation aimed at understanding typical and atypical brain development. This special issue highlights recent advances in methodologies such as brain imaging and the 
development of mouse models and cognitive assays, as we begin to understand gene function in the context of brain and behavior. Yet caution must be taken in considering the functionality of polymorphisms, their frequency, and the validity and reliability of the phenotype or trait to be explained.

\section{REFERENCES}

ARnsten, A. F. T. (2000). Through the looking glass: Differential noradrenergic modulation of prefrontal cortical function. Neural Plasticity, 7, 133-146.

Arnsten, A. F. T., Steere, J. C., \& Hunt, R. D. (1996). The contribution of alpha 2-noradrenergic mechanisms of prefrontal cortical cognitive function: Potential significance for attention-deficit hyperactivity disorder. Archives of General Psychiatry, 53, 448-455.

Bartfai, A., Pedersen, N. L., Asarnow, R. F., \& Schalling, D. (1991). Genetic factors for the span of apprehension test: A study of normal twins. Psychiatry Research, 38, 115-124.

BAtH, K. G., \& LeE, F. S. (2006). Variant BDNF (Val66Met) impact on brain structure and function. Cognitive, Affective, \& Behavioral Neuroscience, 6, 79-85.

Bellugi, U., WANG, P. P., \& JeRnigan, T. L. (1994). Williams syndrome: An unusual neuropsychological profile. In S. Broman \& J. Grafman (Eds.), Atypical cognitive deficits in developmental disorders: Implications for brain function (pp. 23-56). Hillsdale, NJ: Erlbaum.

Bishop, S. J., Cohen, J. D., Fossella, J., Casey, B. J., \& Farah, M. J. (2006). COMT genotype influences prefrontal response to emotional distraction. Cognitive, Affective, \& Behavioral Neuroscience, 6, 62-70.

Brown, S. M., \& HARIRI, A. R. (2006). Neuroimaging studies of serotonin gene polymorphisms: Exploring the interplay of genes, brain, and behavior. Cognitive, Affective, \& Behavioral Neuroscience, 6, 44-52.

Cannon, T. D., Huttunen, M. O., Lönnevist, J., TuulioHenriksson, A., Pirkola, T., Glahn, D., et al. (2000). The inheritance of neuropsychological dysfunction in twins discordant for schizophrenia. American Journal of Human Genetics, 67, 369-382.

Cheverud, J., Falk, D., Vannier, M., Königsberg, L., Helmkamp, R., \& Hildebolt, C. (1990). Heritability of brain size and surface features in rhesus macaques (Macaca mulatta). Journal of Heredity, 81, 51-57.

Corbo, R. M., \& SCACCHI, R. (1999). Apolipoprotein E (APOE) allele distribution in the world. Is APOE*4 a "thrifty" allele? Annals of Human Genetics, 63, 301-310.

Cornblatt, B. A., Risch, N. J., Faris, G., Friedman, D., \& ErLenmeyerKimling, L. (1988). The Continuous Performance Test, identical pairs version (CPT-IP): I. New findings about sustained attention in normal families. Psychiatry Research, 26, 223-238.

Espeseth, T., Greenwood, P. M., Reinvang, I., Fuell, A. M., WalHOVD, K. B., WeSTLYE, L. T., ET AL. (2006). Interactive effects of APOE and CHRNA4 on attention and white matter volume in healthy middle-aged and older adults. Cognitive, Affective, \& Behavioral Neuroscience, 6, 31-43.

Fan, J., Wu, Y., Fossella, J. A., \& Posner, M. I. (2001). Assessing the heritability of attentional networks. BMC Neuroscience, 2, 14.

Fossella, J., Green, A. E., \& FAn, J. (2006). Evaluation of a structural polymorphism in the ankyrin repeat and kinase domain containing-1 (ANKK1) gene and the activation of executive attention networks. Cognitive, Affective, \& Behavioral Neuroscience, 6, 71-78.

GeYer, M. A., \& BrafF, D. L. (1987). Startle habituation and sensorimotor gating in schizophrenia and related animal models. Schizophrenia Bulletin, 13, 643-668.

Hyman, S. E., \& Fenton, W. S. (2003). Medicine: What are the right targets for psychopharmacology? Science, 299, 350-351.

Karmiloff-Smith, A. (2006). The tortuous route from genes to behavior: A neuroconstructivist approach. Cognitive, Affective, \& Behavioral Neuroscience, 6, 9-17.
Katsanis, J., Taylor, J., Iacono, W. G., \& Hammer, M. A. (2000). Heritability of different measures of smooth pursuit eye tracking dysfunction: A study of normal twins. Psychophysiology, 37, 724-730.

KruZich, P. J., Mitchell, S. H., Younkin, A., \& Grandy, D. K. (2006). Dopamine $\mathrm{D}_{2}$ receptors mediate reversal learning in male C57BL/6J mice. Cognitive, Affective, \& Behavioral Neuroscience, 6, 86-90.

Myles-Worsley, M., \& CoON, H. (1997). Genetic and developmental factors in spontaneous selective attention: A study of normal twins. Psychiatry Research, 71, 163-174.

Nachman, M. W., \& Crowell, S. L. (2000) Estimate of the mutation rate per nucleotide in humans. Genetics, 156, 297-304.

Oppenheim, J. S., Skerry, J. E., Tramo, M. J., \& Gazzaniga, M. S. (1989). Magnetic resonance imaging morphology of the corpus callosum in monozygotic twins. Annals of Neurology, 26, 100-104.

Pardo, P. J., Knesevic, M. A., Vogler, G. P., Pardo, J. V., Towne, B. Cloninger, C. R., \& Posner, M. I. (2000). Genetic and state variables of neurocognitive dysfunction in schizophrenia: A twin study. Schizophrenia Bulletin, 26, 459-477.

Rossen, M. L., Jones, W., Wang, P. P., \& Klima, E. S. (1995). Face processing: Remarkable sparing in Williams syndrome. Genetic Counseling, 6, 138-140.

Siest, G., Pillot, T., Regis-Bailly, A., Leininger-Muller, B., SteinMEtZ, J., Galteau, M. M., \& Visvikis, S. (1995). Apolipoprotein E: An important gene and protein to follow in laboratory medicine. Clinical Chemistry, 41, 1068-1086.

Thompson, P. [M.], Cannon, T. D., \& Toga, A. W. (2002). Mapping genetic influences on human brain structure. Annals of Medicine, 34, 523-526.

Thompson, P. M., Giedd, J. N., Woods, R. P., MacDonald, D., Evans, A. C., \& ToGA, A. W. (2000). Growth patterns in the developing brain detected by using continuum mechanical tensor maps. Nature, 404, 190-193.

Tramo, M. J., Loftus, W. C., Stukel, T. A., Green, R. L., Weaver, J. B., \& Gazzaniga, M. S. (1998). Brain size, head size, and intelligence quotient in monozygotic twins. Neurology, 50, 1246-1252.

Waldman, I. D., NigG, J. T., Gizer, I. R., Park, L., Rappley, M. D., $\&$ FrIDERICI, K. (2006). The adrenergic receptor 2- $\alpha$ gene $(A D R A 2 \alpha)$ and neuropsychological executive functions as putative endophenotypes for childhood ADHD. Cognitive, Affective, \& Behavioral Neuroscience, 6, 18-30.

Wrase, J., Reimold, M., Puls, I., Kienast, T., \& Heinz, A. (2006). Serotonergic dysfunction: Brain imaging and behavioral correlates. Cognitive, Affective, \& Behavioral Neuroscience, 6, 53-61.

\section{NOTES}

1. Additional information about the John Merck Fund Summer Institute can be obtained at www.sacklerinstitute.org/cornell/summerinstitute.

2. A number of websites and databases may be of use to the naive reader. The National Center for Biotechnology Information (NCBI) website (http://www.ncbi.nlm.nih.gov) provides a wide array of bioinformatic databases and data mining tools supported and distributed by multidisciplinary research groups. The National Human Genome Research Institute provides excellent educational resources (www.genome .gov/Education/) that complement these databases. The NCBI site known as the Gene Expression Omnibus (www.ncbi.nlm.nih.gov/geo/) is a gene expression repository. Investigators are able to submit gene expression data in a standardized manner that permits queries across and within specific studies. Information about a specific candidate gene can be obtained through other means such as NCBI searches at the LocusLink site (www.ncbi.nlm.nih.gov/LocusLink) and directly through the genome browser at the University of California at Santa Cruz (www.genome .ucsc.edu). Other mouse resources such as the Gene Expression Nervous System Atlas (GENSAT) (www.gensat.org/) and the Allen Brain Atlas (www.brainatlas.org/) provide user-friendly interfaces that permit investigators to explore gene expression patterns. 